\title{
Effect of Variation of Volume of Injected Water in the Air Sacs of Urinary Catheters on Bladder Spasm After Transurethral Plasmakentic Vaporization of Prostate
}

\author{
Hong Caimei $^{1}$, Guo Xiaoxia ${ }^{1}$, Li Qiufeng ${ }^{2}{ }^{*}$, Yang Qi $^{1}$, Chao Xinghui ${ }^{1}$, Ba Longhong ${ }^{1}$ \\ ${ }^{1}$ Department of Urology, The First Affiliated Hospital of Jinan University, Guangzhou, China \\ ${ }^{2}$ The First Affiliated Hospital, Jinan University, Guangzhou, China
}

Email address:

liqf2020@126.com (Li Qiufeng)

${ }^{*}$ Corresponding author

To cite this article:

Hong Caimei, Guo Xiaoxia, Li Qiufeng, Yang Qi, Chao Xinghui, Ba Longhong. Effect of Variation of Volume of Injected Water in the Air Sacs of Urinary Catheters on Bladder Spasm After Transurethral Plasmakentic Vaporization of Prostate. International Journal of Clinical Urology. Vol. 4, No. 1, 2020, pp. 13-16. doi: 10.11648/j.ijcu.20200401.13

Received: January 9, 2020; Accepted: February 3, 2020; Published: February 12, 2020

\begin{abstract}
Objective We aim to explore variation of the injected water volume in the air sacs of indwelling urinary catheters on bladder spasm and blood urine in patients after transurethral plasmakentic vaporization of prostate (TUPKVP). Methods We included 90 patients who had benign prostatic hyperplasia and received transurethral plasmakentic vaporization of prostate in the First Affiliated Hospital of Jinan University from July 2018 to July 2019. They were averagely randomized into three groups: control group, experimental group A and experimental group B. We injected $30 \mathrm{ml}$ of water into the air sacs of urinary catheters of patients postoperatively. At the first day after operation, the water was reduced to $20 \mathrm{ml}$ in experimental group A, $10 \mathrm{ml}$ in experimental group B, and the water volume in control group remained unchanged. The bladder spasm, blood urine and comfort level in three groups were analyzed comparatively. Results Nursing intervention of reducing injected water volume resulted in a lower incidence of bladder spasm in experimental group B than those in other two groups with $10 \%$ on the day of surgery, $3.3 \%$ at the second day postoperatively and no bladder spasm at the third day after operation. There was a significant difference in the incidence of bladder spasm between the three groups $\left(\chi^{2}=19.449, \mathrm{P}=0.000 ; \chi^{2}=6.469, \mathrm{P}=0.040 ; \chi^{2}=8.314, \mathrm{P}=0.013\right)$. Blood urine was milder in experimental group $\mathrm{B}$ than that in other two groups at the third day postoperatively with significant difference $\left(\chi^{2}=6.796, \mathrm{P}=0.037\right)$. Patients in experimental group $\mathrm{B}$ felt more comfortable than those in other two groups with a rate of comfort up to $70 \%$. There was a significant difference in it between three groups $\left(\chi^{2}=9.600, \mathrm{P}=0.008\right)$. Conclusions For patients who underwent transurethral plasmakentic vaporization of prostate, when continuous bladder irrigation and analgesia are withdrawn, reduction of water volume in the air sacs of indwelling urinary catheters to $10 \mathrm{ml}$ can relieve the constriction of the bladder outlet and rubbing bladder mucosa and prostatic fossa and thus reduce the incidence of bladder spasm and blood urine and increase comfort level.
\end{abstract}

Keywords: Transurethral Plasmakentic Vaporization of Prostate, Indwelling Urinary Catheter, Volume of Air Sac, Nursing Intervention

\section{Introduction}

Prostatic hyperplasia is one of the common male diseases clinically. As the population ages, the incidence of prostatic hyperplasia increases year by year. For the benign prostatic hyperplasia, surgical therapy is required. Currently, transurethral resection of prostate (TURP) is gold standard of treatment with advantages of short operation time, light surgical trauma and quick recovery $[1,2]$. However, transurethral resection of prostate is followed by high incidence of transurethral resection syndrome such as water intoxication. Renovation of medical equipment bring about bipolar plasmakentic electronic resection system and laser enucleation which reduce the incidence of transurethral resection syndrome. Clinically, transurethral plasmakentic vaporization has been widely adopted to the treatment for 
benign prostatic hyperplasia $[3,4]$.

Oozing of blood from the wound on prostatic fossa may cause blood clot in bladder and that will lead to urinary blocking and further induce bladder spasm. In order to avoid bleeding from the wound on prostatic fossa after TUPKVP, three-chamber catheters are regularly indwelled and injected saline water to give continuous bladder irrigation. According to the color of drainage, whether to press prostatic fossa with air sac of large volume to stop bleeding is decided. However, there is not a unified standard for the volume of injected water in the air sacs of indwelling catheters. In our department, the regular volume of injected water is $30 \mathrm{ml}$. Research shows that increase in volume of injected water raises constriction of neck of bladder and urethra. The increased constriction is very likely to irritate sensory nerves of bladder and thus stimulate parasympathetic nerves which causes bladder contraction, resulting in bladder spasm. Other research also demonstrates that too much injected water in the air sac causes severe bearing-down feeling and thus repeated micturition desire, causing urethral pain, which is more obvious when patients move $[5,6]$.

To reduce the incidence of bladder spasm induced by indwelling urinary catheters after TUPKVP, the volume of injected water into the air sacs of urinary catheters of 90 patients who received TUPKVP was changed from July 2018 to July 2019 to investigate how the change of injected water in the air sacs of indwelling urinary catheters affects bladder spasm and blood urine. The results are reported as follows.

\section{Data and Methods}

\subsection{General Data}

We enrolled 90 patients who were diagnosed with benign prostatic hyperplasia and admitted to our department from July 2018 to July 2019 as participants. The inclusion criteria are: having not been given muscarinic receptor blocking drugs perioperatively, having indications of surgical treatment for prostatic hyperplasia, being willing to participate in the current research, having provided their informed consents. The 90 patients were averagely randomized into three groups: control group, experimental group A and experimental group B. The patients in control group age from 58 to 87 with an average age of $73.3 \pm 8.4$; patients in experimental group A age from 57 to 86 with an average age of $72.0 \pm 8.2$ and experimental group B from 56 to 86 with an average age of 73.0 18.0 . In the control group. the volume of prostate ranges from $45.1 \mathrm{ml}$ to $105.8 \mathrm{ml}(75.2 \pm 30.5 \mathrm{ml}$ averagely); there are 4 cases of combined diabetes, 6 cases of hypertension, 3 cases of coronary heart diseases; and the duration of indwelling urinary catheters postoperatively is $5.0 \pm 1.2$ days. In the experimental group A, the volume of prostate is $46.3-108.2 \mathrm{ml}$ (with an average of $77.2 \pm 31.4 \mathrm{ml}$ ); there are 5 cases of combined diabetes, 6 cases of hypertension and 2 cases of coronary heart diseases; and the duration of indwelling urinary catheters is $5.1 \pm 1.1$ days. In the experimental group B, the volume of prostate is $45.5-106.9 \mathrm{ml}(76.9 \pm 31.1$ averagely); there are 6 cases of combined diabetes, 4 cases of hypertension and 4 cases of coronary heart diseases; and the duration of indwelling urinary catheters is $4.8 \pm 0.9$ days. There is no significant difference in the age, comorbidity, volume of prostate and other aspects between the three groups ( $>00.05)$. Thus, the comparison of the three groups is worth exploring. The postoperative pathology indicates prostatic hyperplasia.

\subsection{Methods}

Patients in three groups were given transurethral plasmakentic vaporization of prostate after receiving combined spinal-epidural anesthesia. Postoperatively, the same type of F18 three-chamber silica gel urinary catheters (Dalian Kuliaite Medical Products Co. Ltd, China.) were indwelled and $30 \mathrm{ml}$ of water was injected into the air sacs of the urinary catheters. The catheters were pulled outwards to give continuous bladder irrigation and pump for analgesia was used for continuous pain killing. At the first day postoperatively the continuous bladder irrigation was withdrawn and there was no active bleeding during two hours of observation. We reduced the volume of water injected into the air sacs of urinary catheters to $20 \mathrm{ml}$ and $10 \mathrm{ml}$ in experimental group A and experimental group B respectively while the volume of injected water in the control group remained $30 \mathrm{ml}$, and withdrew analgesia pump in three groups. We observed the incidence of bladder spasm and blood urine in the three groups on the day of intervention, and at the second and third day after nursing intervention.

\subsection{Comparative Analysis of Bladder Spasm Score, Blood Urine and Comfort Level Between the Three Groups}

\subsubsection{Bladder Spasm Score}

Any one of precipitant urination, precipitant defecation, painful bladder and no obvious change of drainage color deserves one score; unbearable pain in bladder, urine spillover around urinary catheters and obvious red color of the drainage are two scores separately. When the total score reaches 4 or over 4 , the condition is seen as bladder spasm. If all the above symptoms occur, the condition is 10 score (We referred to the visual analogue scales. The score ranges from 0 to 10 and is in proportion to spasm condition.) $[7,8]$.

\subsubsection{Evaluation of Blood Urine}

Blood urine induced by diseases other than TUPKVP was excluded. The patients were instructed to avoid eating foods and drugs that could cause color change of the urine. We used urine colorimetric card to determine and compare the color of urine between three groups. Red urine including light red, bright red and dark red urine is seen as blood urine.

\subsubsection{Visual Analogue Scales for Evaluation of Comfort Level}

The incidence and degree of pain was evaluated based on the feeling of patients. When urinary catheters are indwelled and there is no frequent, urgent and painful urination, the comfort level is 0 . When there is frequent, urgent urination and urination desire, and the pain score ranges from 1-3, 4-7 
and 8-10, the comfort level is I, II and III respectively [8]. Hence, the level of 0 represents comfortable. The higher the level, the less comfortable it indicates.

\subsection{Statistical Methods}

We used SPSS 17.0 for statistical analysis and the data were shown in the form of (mean \pm standard deviation). Enumeration data were tested by $\chi^{2}$ and $\mathrm{p}<0.05$ indicates significant difference.

\section{Results}

\subsection{Comparison of Incidence of Bladder Spasm and Blood Urine Condition Between Three Groups}

At the first day postoperatively, when changing the volume of injected water into the air sacs of three-chamber catheters, we did not find the catheters fell off. Furthermore, we found the incidence of bladder spasm in the experimental group B was lower than those in other two groups. Besides, patients in experimental group B reported that they felt obviously relieved in the lower abdomen as soon as the volume of the air sacs of the catheters was changed. The incidence of bladder spasm in the experimental group B was $10 \%, 3.3 \%$ and $0 \%$ respectively on the day of operation, at the second and third day after operation. There was a significant difference between the incidences of three days $\left(\chi^{2}=19.449, \mathrm{P}=0.000\right.$; $\left.\chi^{2}=6.469, \mathrm{P}=0.040 ; \chi^{2}=8.314, \mathrm{P}=0.013\right)$. The condition of blood urine in experimental group $\mathrm{B}$ was better than that in experiment group $\mathrm{A}$ and control group and there was a significant difference between three groups $\left(\chi^{2}=6.796\right.$, $\mathrm{P}=0.037)$. The results are shown in Table 1 .

Table 1. Incidence of Bladder Spasm and Blood Urine Condition in the Three Groups after Nursing Intervention (\%).

\begin{tabular}{|c|c|c|c|c|c|c|c|}
\hline \multirow{2}{*}{ Group } & \multirow{2}{*}{$\mathbf{N}$} & \multicolumn{2}{|c|}{ The day of operation } & \multicolumn{2}{|l|}{ Second day } & \multicolumn{2}{|l|}{ Third day } \\
\hline & & Bladder spasm & Blood urine & Bladder spasm & Blood urine & Bladder spasm & Blood urine \\
\hline Control group & 30 & $9(30.0)$ & $11(36.7)$ & $8(26.7)$ & $7(23.3)$ & $7(23.3)$ & $6(20.0)$ \\
\hline Experimental group A & 30 & $5(16.7)$ & $6(20.0)$ & $4(13.3)$ & $5(16.7)$ & $4(13.3)$ & $3(10.0)$ \\
\hline Experimental group B & 30 & $3(10.0)$ & $4(13.3)$ & $1(3.3)$ & $1(3.3)$ & $0(0.00)$ & $0(0.00)$ \\
\hline$\chi^{2}$ & & 19.449 & 4.845 & 6.469 & 5.284 & 8.314 & 6.796 \\
\hline$P$ & & 0.000 & 0.115 & 0.040 & 0.072 & 0.013 & 0.037 \\
\hline
\end{tabular}

\subsection{Comparison of Comfort Level on the Indwelling Catheters Between Three Goups}

After the injected water volume in the air sacs of three-chamber catheters was changed, the comfort level of patients in experimental group B reached $70 \%$ which is significantly higher than the $50 \%$ and $30 \%$ in experimental group $\mathrm{A}$ and control group respectively. There was a significant difference between three groups $\left(\chi^{2}=9.600\right.$, $\mathrm{p}=0.008$ ) as shown in Table 2 .

Table 2. Comparison of Comfort Level on the Indwelling Catheters between Three Groups (\%).

\begin{tabular}{|c|c|c|c|c|c|}
\hline \multirow{2}{*}{ Group } & \multirow{2}{*}{$\mathbf{N}$} & \multicolumn{4}{|c|}{ Comfort level } \\
\hline & & $\mathbf{0}$ & I & II & III \\
\hline Control group & 30 & $9(30.0)^{*}$ & $10(33.3)$ & $9(30)$ & $2(6.7)$ \\
\hline Experimental group A & 30 & $15(50.0)$ & $8(26.7)$ & $6(20.0)$ & $1(3.3)$ \\
\hline Experimental group $\mathrm{B}$ & 30 & $21(70.0)$ & $6(20.0)$ & $3(10)$ & $0(0.0)$ \\
\hline$\chi^{2}$ & & 9.600 & & & \\
\hline$P$ & & 0.008 & & & \\
\hline
\end{tabular}

Notice: * indicated that compared with experimental group A and B, there was a significant difference $(\mathrm{P}<0.05)$.

\section{Discussion}

Complications of transurethral plasmakentic vaporization of prostate include bladder spasm, bleeding, urinary incontinence, urinary tract infection, etc. [2, 9-10]. Bladder spasm may aggravate prostate wound bleeding and the blood clot would block the ureter. Inadequate drainage of urine in return will induce bladder spasm, causing physical pain to patients and damaging their physical and mental health and postoperative recovery. The volume of injected water in the air sacs of indwelling catheters after TUPKVP should function to fix the urinary catheters and the adverse reaction the catheters cause should be reduced as much as possible. If too much water is injected into the air sacs, the neck of bladder will be constricted and patients will have bearing-down feeling in the lower abdomen, very likely to cause bladder spasm. Besides, the larger the air sacs are, the more severe the friction between air sacs and mucosa of prostatic fossa is, which means the bleeding is very likely to occur. However, if the volume of injected water is too small, the comfort level may be higher but the urinary catheters are more likely to fall off.

Clinically, there is not a standard volume of injected water into the air sacs. That is mainly because it is hard to decide a standardized volume that is applicable to all populations [11-13]. The volume should be decided according to sex, age, diseases and other factors [14-16]. Some research [16] show there should be a difference in the volume of injected water between females and males and suggest that the best volume for males whose operation is not TUPKVP is 7-10 ml. Nevertheless, TUPKVP expands internal urethral orifice. If the volume of injected water is too small, the urinary catheters are likely to fall off. Some researchers explain that when the 
injected water is not enough, the air sac is not expanded enough. Hence, when subject to external force or the patients' abdominal pressure raises, air sacs are easily deformed and the deformation causes falling off of urinary catheters. Therefore, the volume of injected water in the air sacs of urinary catheters is very important to the treatment and comfort level for patients $[6,11,16]$.

In the current research, we changed the volume of injected water in the three-chamber urinary catheters in the experimental group B at the first day after operation. We found that the incidence of bladder spasm and blood urine in the experimental group B was lower and the comfort level was higher than that in the control group and experimental group A Besides, we found that there was no external force and abdominal pressure increase-induced urinary catheter falling off. Instead, patients in experimental group B reported that they felt relieved in lower abdomen and there was no severe discomfort with urinary catheters. Patients in control group complained that they had bearing-down feeling in lower abdomen, precipitant urination, urine spillover, red urine or dark red urine most of time when they performed daily activities with the urinary catheters. Patients in experimental group B complained the same symptoms as patients in control group while their symptoms were much milder than those of patients in control group. The discomfort that patients in control group and experimental group A complain may be because the large air sacs of urinary catheters are stuck in the prostatic fossa which compresses the neck of bladder and rubs the wound of operation due to gravity when patients are standing. The bigger the air sacs are, the larger the rubbing surface between air sacs and mucosa of bladder is and the stimulation to nerves in the inner wall of the bladder increases. That means damage to mucosa of bladder increases, so patients feel more painful and uncomfortable and the incidence of blood urine is higher.

\section{Conclusions}

In conclusion, at the first day after TUPKVP when continuous bladder irrigation and analgesia are withdrawn, reduction of volume of injected water in the air sacs of indwelling urinary catheters to $10 \mathrm{ml}$ reduces constriction to bladder outlet and rubbing to bladder mucosa and prostatic fossa. As a result, within three days postoperatively, the incidence of bladder spasm and blood urine decreases significantly. Therefore, the intervention of reducing the volume of injected water can relieve patients' discomfort and is beneficial to their recovery, and thus is well worth clinical application and promotion.

\section{References}

[1] Rassweiler, J., Teber, D., Kuntz, R., \& Hofmann, R. (2006). Complications of transurethral resection of the prostate (turp)-incidence, management, and prevention. European Urology, 50 (5), 969-980.
[2] May, A., Broggi, E., Lorphelin, H., Tabchouri, N., \& Bruyere, F. (2014). Comparison of the risk of postoperative infection between transurethral vaporesection and transurethral resection of the prostate. Lasers in Surgery and Medicine, 46 (5), 405-11.

[3] Taylor, B. L., \& Jaffe, W. I. (2015). Electrosurgical transurethral resection of the prostate and transurethral incision of the prostate (monopolar techniques). The Canadian Journal of Urology, 22 (5S1), 24-29.

[4] Sio, M. D., Autorino, R., Quarto, G., Damiano, R., Sisto Perdonà, \& Lorenzo, G. D., et al. (2006). Gyrus bipolar versus standard monopolar transurethral resection of the prostate: a randomized prospective trial. Urology, 67 (1), 69-72.

[5] Dybowski, B. A., Piotr Zapała, Bres-Niewada, E., Łukasz Zapała, \& Radziszewski, P. (2018). Catheter-associated bacterial flora in patients with benign prostatic hyperplasia: shift in antimicrobial susceptibility pattern. BMC Infectious Diseases, 18 (1), 590.

[6] Starkman, J. S., \& Santucci, R. A. (2005). Comparison of bipolar transurethral resection of the prostate with standard transurethral prostatectomy: shorter stay, earlier catheter removal and fewer complications. BJU International, 95 (1): 69-71.

[7] Gillies, D., Lane, L., Murrell, D., \& Cohen, R. (2003). Bladder spasm in children after surgery for ureteric reimplantation. Pediatric Surgery International, 19 (11), 733-736.

[8] Hammadeh, M. Y., \& Philp, T. (2003). Transurethral electrovaporization of the prostate (tuvp) is effective, safe and durable. Prostate Cancer Prostatic Dis, 6 (2), 121-126.

[9] Geavlete, P. (2010). Is classical transurethral resection of the prostate, the gold standard endoscopic treatment for benign prostate hyperplasia, in real danger of being replaced? European Urology, 58 (3), 356-358.

[10] Emerson Luís Zani, \& Netto, N. R. (2007). Is the minimally invasive treatment as good as transurethral resection for benign prostatic hyperplasia? International Urology and Nephrology, 39 (1), 161-168.

[11] Robinson, \& John. (2005). Changing indwelling urinary catheters using bladder infill. British Journal of Community Nursing, 10 (6), 266-269.

[12] Chander, J, Vanitha, V., Lal, P., \& Ramteke, V. K. (2003). Transurethral resection of the prostate as catheter-free day-care surgery. BJU International, 92 (4), 422-425.

[13] Wilson, I. D., Bramwell, S. P., \& Hollins, G. W. (2000). A randomized trial comparing bladder infusion with standard catheter removal after transurethral resection of the prostate. BJU International, 86 (9), 993-995.

[14] Pomfret, \& Ian. (2007). Urinary catheterization: selection and clinical management. British Journal of Community Nursing, $12(8), 348-354$.

[15] Taylor, P. (2002). Management of continence and urinary catheter care. Journal of Clinical Nursing, 11 (7), 770-770.

[16] Robinson, \& John. (2006). Selecting a urinary catheter and drainage system. British Journal of Nursing, 15 (19), 1045-1050. 